\title{
Effects of Low-Frequency Biasing on Otoacoustic and Neural Measures Suggest that Stimulus-Frequency Otoacoustic Emissions Originate Near the Peak Region of the Traveling Wave
}

\author{
JEFFERY T. LICHTENHAN ${ }^{1,2}$ \\ ${ }^{1}$ Massachusetts Eye E Ear Infirmary, Eaton-Peabody Laboratory of Auditory Physiology, 243 Charles Street, Boston, MA \\ 02114, USA \\ ${ }^{2}$ Department of Otology and Laryngology, Harvard Medical School, Boston, MA 02115, USA
}

Received: 23 April 2011; Accepted: 29 September 2011; Online publication: 15 October 2011

\begin{abstract}
Stimulus-frequency otoacoustic emissions (SFOAEs) have been used to study a variety of topics in cochlear mechanics, although a current topic of debate is where in the cochlea these emissions are generated. One hypothesis is that SFOAE generation is predominately near the peak region of the traveling wave. An opposing hypothesis is that SFOAE generation near the peak region is deemphasized compared to generation in the tail region of the traveling wave. A comparison was made between the effect of low-frequency biasing on both SFOAEs and a physiologic measure that arises from the peak region of the traveling wave-the compound action potential (CAP). SFOAE biasing was measured as the amplitude of spectral sidebands from varying bias tone levels. CAP biasing was measured as the suppression of CAP amplitude from varying bias tone levels. Measures of biasing effects were made throughout the cochlea. Results from cats show that the level of bias tone needed for maximum SFOAE sidebands and for 50\% CAP reduction increased as probe frequency increased. Results from guinea pigs show an irregular bias effect as a function of probe frequency. In both species, there was a strong and positive relationship between the bias level needed for maximum SFOAE sidebands and for $50 \%$ CAP suppression. This relationship is consistent with the

Correspondence to: Jeffery T. Lichtenhan - Department of Otology and Laryngology $\cdot$ Harvard Medical School - Boston, MA 02115, USA. Telephone: $\pm 1-617-573-3745$; fax: $\pm 1-617-720-4408$; email: jlichtenhan@gmail.com
\end{abstract}

hypothesis that the majority of SFOAE is generated near the peak region of the traveling wave.

Keywords: amplitude modulation, low-frequency bias tone, cochlea, basilar membrane, auditory nerve, compound action potential, otoacoustic emissions

\section{INTRODUCTION}

Otoacoustic emissions are acoustical signals recorded non-invasively from the outer ear canal that arise from cochlear amplification. Stimulus-frequency otoacoustic emissions (SFOAEs) are the simplest otoacoustic emission to interpret, as they arise from stimulation with a single pure-tone of low to moderate level. SFOAEs are less commonly used, however, because of signal processing difficulties involved with recording this emission that occurs during the same time and at the same frequency as the stimulus. Nevertheless, it seems as though the relatively simple nature of SFOAEs makes them suitable, and perhaps ideal, for non-invasively studying a variety of cochlear mechanics. SFOAEs from humans and animals have been used to understand how emissions propagate out of the cochlea (Shera et al. 2008; Meenderink and van der Heijden 2010), to characterize the sharpness of cochlear tuning (Shera et al. 2002; Shera and Guinan 2003; Schairer et al. 2006; Bentsen et al. 2011), and to 
test models of emission generation (Moleti and Sisto 2003; Konrad-Martin and Keefe 2003, 2005; Moleti et al. 2005; Sisto and Moleti 2007; Moleti and Sisto 2008).

Despite the progress of the aforesaid studies, controversy proposes that the relation between empirical SFOAEs and direct measures of cochlear mechanics are more complicated than that suggested by theoretical-based models. A theoretical model by Zweig and Shera (1995) suggested that SFOAEs generation is near the peak region of the traveling wave. Empirical results from Brass and Kemp (1993) and Keefe et al. (2008) who used classic two-tone suppression paradigms showed that SFOAEs behave as though they originate primarily near the peak region of the traveling wave-the least amount of suppressor level was needed for criterion suppression when the frequency of the suppressor tone was nearest, not greater than, the probe tone frequency. In contrast, Siegel et al. (2005) compared empirical forward propagation time from basilar membrane vibrations and neural measures to reverse propagation time from SFOAE group delay measures and hypothesized that SFOAE generators are widely distributed along the cochlea though "...sources should undergo spatial summation in the basal (long-wavelength) cochlear regions, and strong cancellation near the peak or apical (short-wavelength) region of the forward BM traveling wave, thus deemphasizing [peak region] contributions and emphasizing [tail region] contributions" [p. 2442 of Siegel et al. (2005)].

There are ways to advance the previous empirical efforts that aimed to determine where SFOAEs are generated. Siegel et al.'s (2005) measures of forward propagation time were obtained from one group of animals and compared to measures of reverse propagation time obtained from a different group of animals. Since there is high inter- and intra-ear variability in SFOAE and single-fiber measures used by Siegel et al. (2005) (e.g., figure 4 of Siegel et al. 2005; figure 5 of Liberman 1984; figure 8 of Liberman 1990), making comparisons between otoacoustic and neural measures might best be done on an ear-by-ear basis.

The goal of the current study is to understand where SFOAEs are generated. Our strategy is to compare the effect of low-frequency biasing on SFOAEs and on auditory nerve compound action potentials (CAPs) from the same animals, at the same probe frequencies, both recorded closely in time. For low-level, high-frequency stimuli, CAPs originate from a narrow cochlear region about the stimuli's characteristic frequency place. Evidence for this well-established understanding is that CAP latency is similar to single-fiber post-stimulus time histogram peak latency (Kiang 1965), CAP suppression tuning curves are similar to single-fiber tuning curves (Dolan et al. 1985; Cheatham et al. 2010), and lesion studies (Cody et al.
1980). In biasing experiments, a high-level, lowfrequency tone is presented simultaneously with a higher frequency probe tone. Low-frequency biasing positions the outer hair cell mechano-electric transducer channels into nonlinear operating regions - an action that reduces cochlear amplifier gain, amplitude modulates SFOAEs, and suppresses CAP responses. If SFOAEs and CAPs originate from a similar cochlear region, they should be similarly influenced by lowfrequency biasing. If, on the other hand, SFOAEs from the peak region generators are "deemphasized" more than those from the tail regions, the effect of low-frequency biasing should be different between SFOAEs and CAPs. The results of the experiments offer empirical evidence for the hypothesis that SFOAEs are dominated by generation near the peak region of the traveling wave.

\section{METHODS}

\section{Model: a guide to physiologic experimental expectations}

Results from a simple model are presented in Figure 1 before experimental results are presented in order to guide readers' expectations for physiologic SFOAE and CAP biasing presented later. A sigmodial saturating nonlinear function modeling hair cell transduction, such as that from Hudspeth and Corey (1977) or Géléoc et al. (1997), is commonly used to represent mechano-electric transduction in vivo (e.g., Weiss and Leong 1985; Chertoff et al. 2003; Salt et al. 2009). Here we used

$$
\tanh (x) \text { for } x<0 \text {, and } \tanh (R * x) / R \text { for } x>0
$$

where tanh is a hyperbolic tangent function, $x$ is mechanical drive, and $R$ is what we call the "ratio of asymptotes". $R$ was 2.5 for the illustration of Figure 1 . This model transducer function can be thought of as a composite estimate of mechano-electric transducer functions from individual stereocilia bundles across various cochlear segments about a moderate-level pure tone's cochlear characteristic frequency place. Although the model transducer function is rather simple, it produced the salient features of physiologic SFOAE sideband growth and CAP suppression presented below in the "Results" section. The model transducer function is not intended to explain SFOAE generation; rather, its intended use is to help explain biasing experiments.

The first-order sidebands from the model simulations were defined as the energy in the spectral bins centered at the probe frequency \pm the bias tone frequency. The probe tone level was held constant 


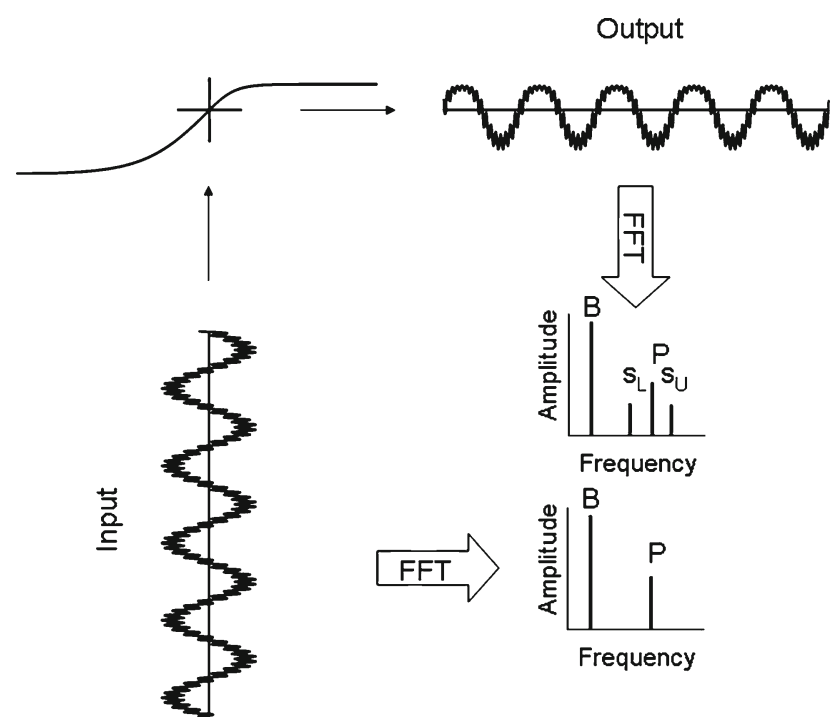

FIG. 1. The essence of our modeling efforts for one level of a lowfrequency bias tone. In the lower left, a low-frequency bias tone and higher frequency probe tone are shown as mechanical input to the model transducer function in the upper left. The model transducer function represents the outer hair cell input-output function with mechanical drive on the $x$-axis and outer hair cell current on the $y$ axis. The output in the upper right shows distortion in the time domain. The lower right illustrates that the output in the frequency domain has a bias tone $(B)$, probe tone $(P)$, and lower and upper sidebands $\left(s_{L}\right.$ and $\left.s_{U}\right)$. In contrast, the input has no sidebands.

and the level of the low-frequency bias tone was varied. As the level of the low-frequency bias tone was increased, amplitude modulation of the probe tone increased (Fig. 2). Further bias tone level increases yielded a decline in sideband amplitude. (Lower and upper sidebands from the model were of similar amplitude, and thus we plotted only the lower side-

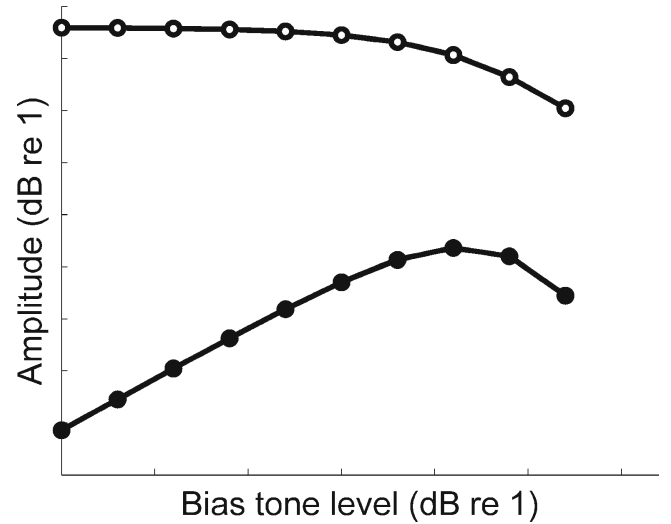

FIG. 2. Amplitude of modeled sidebands (filled symbols) and probe output (open symbols) as a function of bias tone level. The sidebands resulted from amplitude modulation of the probe tone. The sideband growth was governed by the gain, or slope, of the transducer function. The decline of the sidebands, as well as the probe tone at the output, was also governed by the morphology of the transducer function. This is a guide to our interpretation of our physiologic SFOAE biasing experiment described below. band.) The increasing and decreasing sideband amplitude from this modeling effort explain why, as shown below, physiologic SFOAE sidebands increased, and then decreased, as bias level was increased. At low bias levels, there is enough modulation of nonlinear cochlear amplification at the probe frequency to produce sidebands. As the level of the low-frequency tone increases, maximum sidebands result because outer hair cell stereocilia are biased into the saturation regions of their input-output functions. Further increases in bias tone level results in a reduction of overall basilar membrane response to the probe tone and, as a consequence, a decline of sideband amplitude. The maximum sideband amplitude thus indicates the bias level needed for the greatest achievable amount of first-order cochlear amplifier gain modulation.

Figure 3 shows the slope of the model transducer function throughout one cycle of low-frequency biasing for a variety of bias tone levels. The amount of suppression was asymmetric during the bias cycle because the model transducer function of Figure 1 was asymmetric about its origin. As the level of the low-frequency bias tone increased, the probe was positioned into low-slope regions of the transducer curve. The model output behavior in Figure 3 is an interpretative guide to our physiologic CAP suppression experiments shown below. As we will see later on, throughout one bias cycle the low-frequency tone twice positioned the probe tone into low-slope regions of the in vivo transducer function and suppressed the tone-pip evoked CAPs. No suppression occurred when there was no bias tone sound pressure level (i.e., at $0^{\circ}$, $180^{\circ}$, and $360^{\circ}$ ), showing that the slope of the in vivo transducer function directly influenced the peak-topeak amplitude of the CAP.

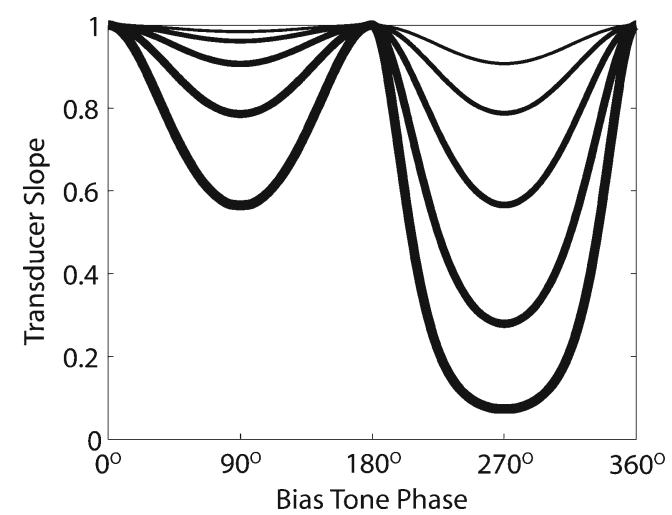

FIG. 3. The slope of the model transducer function throughout one cycle of the low-frequency bias tone. Twice each biasing cycle, the gain of the probe tone was suppressed. Amount of suppression was bias level-dependent. Line thickening represents increasing bias tone level. This guides our interpretation of physiologic CAP biasing experiments shown below: since cochlear amplifier gain depends on the slope of the transducer function, a variation of cochlear amplifier suppression produces varying amounts CAP amplitude reduction. 


\section{Physiologic stimuli and recordings}

All stimulus calibration, stimulus generation, and data acquisition were performed with data-acquisition boards [National Instruments (PXI 4461 24-bit Dynamic Signal Analyzer and PXI 6221 16-bit Mseries)] controlled with custom written software in LabVIEW. Bias tones of $50 \mathrm{~Hz}$ were delivered to cat ears with a dynamic headphone [Beyerdynamic (DT48)] while all other stimuli were delivered with a 1-in. condenser earphone [Bruel \& Kjaer (B\&K 4145)]. The same DT48 earphone was used to deliver bias tones of $150 \mathrm{~Hz}, 70 \mathrm{~Hz}$, or $50 \mathrm{~Hz}$ to guinea pig ears while all other stimuli were delivered with two 15mm dynamic speakers [CUI, Inc. (CDMG15008-03)]. A 40-dB SPL probe tone was used for all SFOAE and CAP biasing measures. Tone-pips for all CAP measures were ramped on and off with a $0.5-\mathrm{ms} \cos ^{2}$ window and had a 0.5 -ms plateau.

Microphones and earphones were housed in a custom made acoustic assembly appropriate for either cat (Kiang 1965) or a modified custom acoustic assembly for guinea pig (http://www.masseyeandear.org/research/ent/ eaton-peabody/epl-engineering-resources/). Cat ear canal acoustics were recorded with a condenser microphone [Knowles (EK-23028-000)]. Guinea pig ear canal signals were recorded with a probe microphone [Etymōtic Research (ER10c)] attached to the probe tube of the custom assembly. Before a suitable microphone was found for cat experiments, several different microphones were tried that (1) would fit into our custom made acoustic assembly housing for cat experiments and (2) could not be biased by our low-frequency tone levels, which would have caused system distortion sidebands. For SFOAE recordings, a sampling frequency of $125 \mathrm{kHz}$ was used, buffer duration was $200.3 \mathrm{~ms}$, and averages were made from 128 presentations. CAP measures were averaged responses from 32 presentations. Tone-pip levels for CAP audiograms were varied until a $10-\mu \mathrm{V}$ peak-to-peak criterion was achieved. All analysis was performed with custom written software in MATLAB.

\section{Procedures}

All procedures were approved by the Institutional Animal Care and Use Committee at the Massachusetts Eye and Ear Infirmary. Cats were anesthetized with pentobarbital and urethane while guinea pigs were anesthetized with pentobarbital, droperidol, and fentanyl. Because their skull was opened for single-fiber experiments not reported here, cats were given dexamethasone to prevent neural swelling and part of the brain was aspirated. All animals were euthanized without recovery at the conclusion of experiments. A cannula was inserted into the trachea of both cats and guinea pigs and, when needed, artificial ventilation maintained end-tidal $\mathrm{CO}_{2}$ levels with a target of $4.5 \%$ to $5 \%$. Body temperature was kept at a mean of $38^{\circ} \mathrm{C}$. Cat and guinea pig soft tissue was removed from dorsal and posterior areas of the skull, both bulla, and ear canals. An opening in the bulla was made to access the round window. Guinea pigs' heads were secured by cementing to an aluminum skull block. Cats' heads were secured by support bars placed under the teeth of the upper jaw, hooks put into orbits, and hollow ear bars that were also used for sound delivery. For both species, a silver wire electrode was placed near the round window for CAP recordings, a screw electrode was put into the skull's vertex for auditory brainstem response (ABR), and a pellet electrode was placed on the exposed neck musculature. For cat experiments only, the skull was opened and the brain was aspirated to expose the auditory nerve.

Auditory status throughout the experiment was monitored with CAP audiograms, click-evoked ABRs, click-evoked CAP level series, and SFOAEs recorded with nonlinear-compression and/or two-tone suppressor paradigms (Kemp and Chum 1980). The probe level was $40 \mathrm{~dB}$ SPL and the suppressor level was $60 \mathrm{~dB}$ SPL for both nonlinear-compression and twotone suppressor paradigms. The suppressor frequency was at the probe frequency for the compression paradigm and was $50 \mathrm{~Hz}$ higher than the probe frequency for the two-tone suppressor paradigm. To find a suitable frequency region for SFOAE and CAP biasing, SFOAEs were measured from $1 \mathrm{kHz}$ to $30 \mathrm{kHz}$ with probe frequencies spanning $\sim 1$ or $2 \mathrm{kHz}$ using $100-\mathrm{Hz}$ to $200-\mathrm{Hz}$ steps that allowed proper phase unwrapping. To expedite this screening procedure, on most occasions two SFOAEs were simultaneously evoked with probe tones spaced anywhere from $5 \mathrm{kHz}$ to $18 \mathrm{kHz}$ apart. A frequency region was deemed usable if average to large ( $\sim 10$ to $20 \mathrm{~dB}$ SPL) SFOAEs could be recorded and if the SFOAE was not near an abrupt change in the phase-versus-frequency plot.

At the usable probe frequencies, both SFOAEs and CAP were low-frequency biased. SFOAEs were biased by presenting a low-frequency tone that was stepped, in most instances, from 84 to $110 \mathrm{~dB}$ SPL and a probe held constant at $40 \mathrm{~dB}$ SPL. Generally, SFOAEs were biased with even number $\mathrm{dB}$ bias levels (e.g., 84, 86, etc...) immediately before CAP biasing measurements and with odd number dB bias levels (e.g., 85, 87, etc...) immediately following CAP biasing measurements. SFOAE biasing generally took about 10 to $15 \mathrm{~min}$ for one probe frequency. At the same frequency as that used for SFOAE biasing, CAPs evoked from $40 \mathrm{~dB}$ SPL tone-pips were biased with low-frequency tones that varied considerably in level. Generally, a few different bias levels were presented, the results were visually inspected, another bias level 
was chosen, and the process repeated until the necessary information for the analysis (described below in the section "CAP analysis") was obtained. Averaged round window electrical activity was recorded from tone-pips placed at 12 different bias tone phases from $0^{\circ}$ to $330^{\circ}$ in $30^{\circ}$ steps, from a repeat recording at $0^{\circ}$ (i.e., $360^{\circ}$ ), from two tone-pips in the absence of the bias tone, and from the bias tone alone in the absence of a tone-pip. The total amount of time needed to collect CAP biasing measures at one tonepip frequency took anywhere from about 10 to 20 min. In-ear sound source calibrations, CAP audiograms, click-evoked CAP level series, click-evoked ABRs, and frequent SFOAE biasing were repeated frequently throughout the experiment.

\section{SFOAE analysis}

Simultaneous presentation of a probe and low-frequency bias tone amplitude modulated the SFOAE evoked by the probe and produced spectral sidebands. These SFOAE sidebands were used as a measure of cochlear amplifier gain modulation. First-order sidebands were at the probe frequency \pm the bias frequency. Smaller, higher-order sidebands [e.g., probe frequency $\pm(2 \times$ bias frequency $)]$ were present in the ear canal signal though were not evaluated in our experiments. The first-order SFOAE sideband levels were determined from the averaged ear canal pressure waveform using Fourier analysis in MATLAB. An SFOAE sideband-bias-level function was constructed by plotting the amplitude of the lower and upper sidebands as a function of bias level for each probe frequency. Both lower (i.e., probe frequency-bias frequency) and upper (i.e., probe frequency+bias frequency) first-order sideband-bias-level functions were fit with a quadratic (polyfitweighted) polynomial and an average quadratic was calculated. The bias level needed for maximum SFOAE sidebands was determined by the maximum of the average quadratic fit. The width of SFOAE sideband growth curve was arbitrarily defined as the range of the bias tone levels at $0.5 \mathrm{~dB}$ down from the maximum.

\section{CAP analysis}

The response from the bias tone presented alone was subtracted from bias tone plus tone-pip responses in order to cancel out cochlear responses from the bias tone that occurred simultaneously with responses of the tone-pip. CAP peak-to-peak amplitudes were measured from the 12 bias tone phases and normalized to the average of the two CAPs amplitudes evoked in the absence of a bias tone. Plots of peakto-peak CAP amplitude measures as a function of bias tone phase were converted to the frequency domain, the first three Fourier components (i.e., the DC along with the first two complex numbers) were kept, and then returned to the time domain. These fits to the CAP amplitude versus bias tone phases were used to estimate the bias levels needed to suppress the CAP by $40 \%, 50 \%$, and $60 \%$. The minima were found and plotted as a function of bias level. These minimumbias level functions were then fit with a second-order polynomial from which the bias levels associated with $40 \%, 50 \%$, and $60 \%$ suppression were estimated.

\section{RESULTS}

\section{Physiologic SFOAE modulation}

Figure 4 shows example spectra of the ear canal sound pressure level from an ear when the cat was alive (top panel) and dead (bottom panel). The live ear produced sidebands because the SFOAE evoked from the probe tone was amplitude modulated by the bias tone. In contrast, the dead ear produced no SFOAE, and thus no sidebands, which is why only the $40 \mathrm{~dB}$ SPL probe and the noise floor are seen in the bottom panel.

Figure 5 shows an example of how the first-order SFOAE sidebands grew, and then decreased, as bias
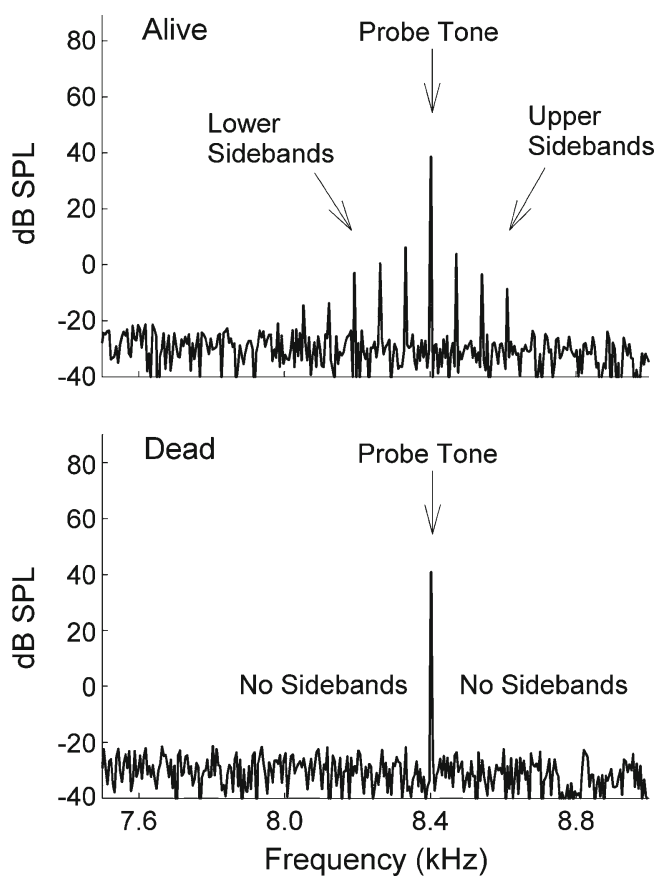

FIG. 4. Example ear canal spectra from when a cat was living (upper panel) and dead (lower panel). For these examples, only one $50 \mathrm{~Hz}$ bias tone level was presented though it is not seen on this scale. The bias tone amplitude modulated the SFOAE generated by the probe tone and produced sidebands visible in the frequency domain. For the experiments reported here, only the first-order sidebands (i.e., probe frequency \pm the bias tone frequency) were considered. 


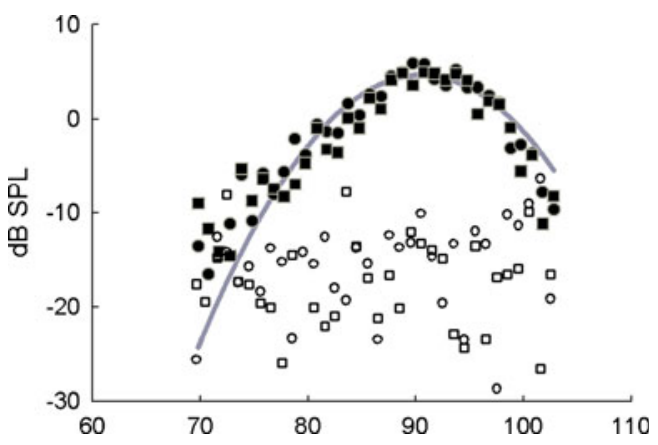

FIG. 5. Example cat SFOAE sidebands as a function of bias level. Amplitudes of lower (i.e., probe tone frequency-bias tone frequency) sidebands from a living animal are represented by filled circles while upper (i.e., probe tone frequency+bias tone frequency) sidebands are represented as filled squares. The unfilled symbols are the corresponding sidebands from a dead animal. The thick gray line is a second-order polynomial fit to the region of maximum SFOAE sidebands. For this example, the probe tone frequency was $2.803 \mathrm{kHz}$ and the bias level producing maximum SFOAE sidebands was $90.8 \mathrm{~dB}$ SPL according to the quadratic fit.

level was increased. The index for the amount of bias level needed to achieve criterion cochlear amplifier gain modulation modulation was the bias tone level which produced the largest SFOAE sideband: the maximum of the sideband-bias-level function.

\section{Physiologic CAP modulation}

Figure 6 shows a series of example CAP modulation curves from one tone-pip frequency from one cat ear. Previous reports investigating the effects of lowfrequency biasing on CAPs used guinea pigs (e.g., Klis and Smoorenburg 1985, 1988; Sellick et al. 1982; Patuzzi et al. 1989) and this is the first report using cat. CAP suppression occurred from the tone-pip being twice positioned throughout the bias cycle into

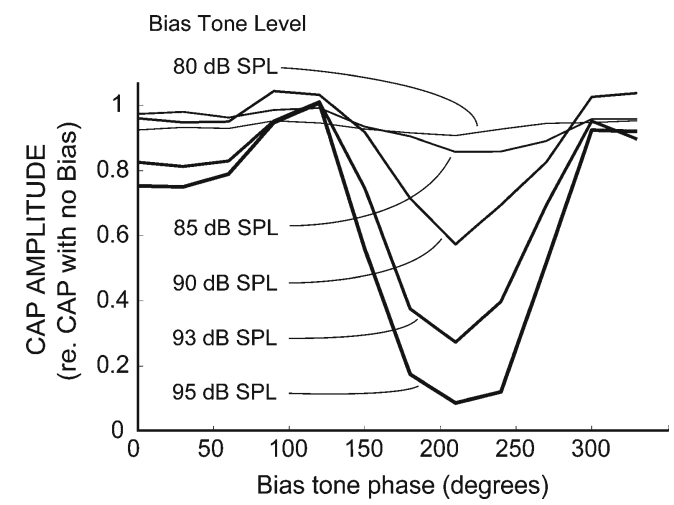

FIG. 6. Example cat CAP amplitude as a function of bias tone phase and level. Lines connect CAP peak-to-peak amplitude measures obtained from different levels and phases of the bias tone. The lines thicken slightly to help illustrate increasing bias level. The amount of CAP suppression varied with the level and phase of the bias tone. For this example, 50\% suppression occurred at a bias tone level of $91 \mathrm{~dB}$ SPL and phase of $210^{\circ}$. The tone-pip frequency was $6.399 \mathrm{kHz}$. low-slope regions of the in vivo transducer function causing reduction of cochlear amplifier gain. As bias level was increased, the suppression of CAP peak-topeak amplitude increased. The largest CAP suppression occurred when tone-pips were presented at specific bias tone phases. Further increases in bias levels beyond those shown in the example in Figure 6 would have eventually produced $100 \%$ suppression during one bias phase and then during both the first and second half of the bias cycle. Increasing bias level beyond the threshold of $100 \%$ suppression would eventually suppress all of the response. We thus avoided high levels. In contrast, slight modulation with low bias levels produced small CAP suppression that could occasionally be difficult to distinguish from no suppression. Thus, $50 \%$ was the clearest, most reliable, index of the influence of low-frequency suppression of the CAP.

\section{SFOAE and CAP modulation as a function of frequency in cat}

The upper panel of Figure 7 shows the bias levels needed to obtain maximum SFOAE sidebands as a function of probe frequency. Data are shown for five ears from four cats. Different symbols indicate measures obtained from different cat ears. A linear regression analysis showed that the error measure did not vary significantly with probe frequency, which means that the width of the function describing
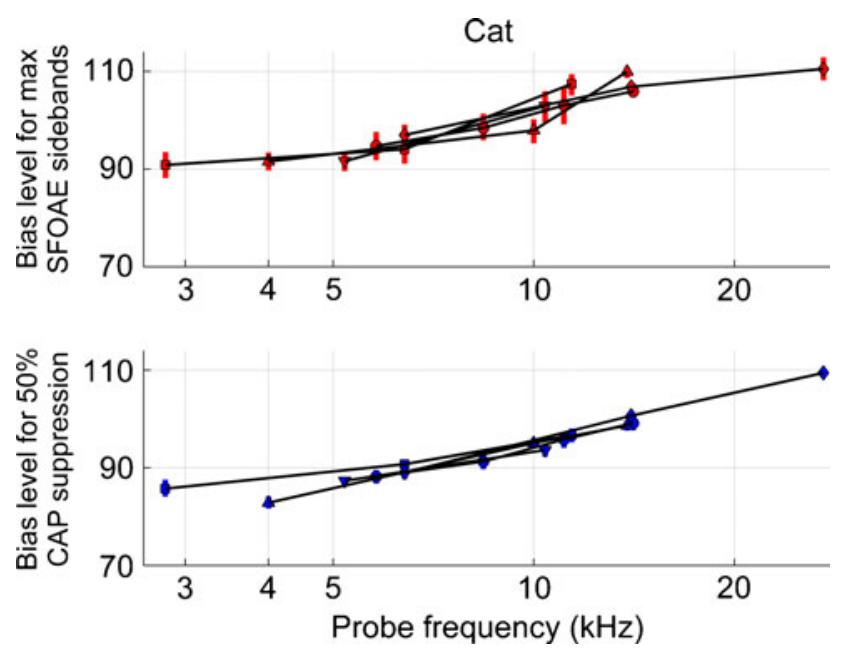

FIG. 7. The upper panel shows the bias level (dB SPL) that produced maximum SFOAE sidebands as a function of probe frequency. Different symbols represent different cat ears. Vertical error bars are the width of the sideband versus bias level curves (e.g., Fig. 2) measured $0.5 \mathrm{~dB}$ down from the maximum. The lower panel shows the bias level (dB SPL) that produced $50 \%$ CAP suppression as a function of frequency. CAP biasing was done at the same probe frequencies as SFOAE biasing. These cat SFOAE and CAP data show a systematic trend that, as compared to lower frequencies, greater bias levels were needed to produce maximum SFOAE sidebands and CAP suppression at higher frequencies. 
sidebands as a function bias level (e.g., Fig. 5) did not vary with frequency. Lines connect measures obtained from different tone frequencies in a given ear. As compared to lower frequency SFOAEs, higher frequency SFOAEs required greater bias levels to achieve maximum modulation. There was little between-ear variability for these cat SFOAE data.

The lower panel of Figure 7 shows the bias tone level that produced 50\% CAP suppression in cat as a function of tone-pip frequency. Different blue symbols indicate measures obtained from different cat ears. The vertical error bars, which are barely noticeable on this scale, though will be strikingly obvious later in Figure 9, are estimates of $40 \%$ and $60 \%$ suppression. Linear regression showed that the error measure did not vary significantly with tone-pip frequency. Lines connect the $50 \%$ suppression measures obtained from different tone-pip frequencies used in a given ear. The CAP tone-pip frequencies were the same as the SFOAE probe frequencies. As tone-pip frequency increased, the level of the low-frequency bias tone needed to achieve 50\% CAP suppression increased. Similar to cat SFOAE data, there was little between-ear variability in the CAP data.

\section{SFOAE and CAP modulation as a function of frequency in guinea pig}

The upper panel of Figure 8 shows the bias level needed to yield maximum SFOAE sidebands in guinea pig as a function of probe frequency. The width of the sideband versus bias level functions did not depend on probe frequency, as a linear regression analysis showed that the error measure did not vary significantly with tone-pip frequency. Compared to the cat SFOAEs data in Figure 7, guinea pig SFOAEs in Figure 8 required lower bias tone levels to yield maximum SFOAE sidebands. Also unlike cat data, there was considerable variability across probe frequency and ears. A Levene's test of homoscedasticity showed that the variance of the SFOAE data from $50 \mathrm{~Hz}$ and $70 \mathrm{~Hz}$ biasing did not differ $(p=0.0702)$, which is why these data are plotted in the same color and grouped together for the remainder of this report. The variance of the data obtained with a $150 \mathrm{~Hz}$ bias tone was different from that obtained with the lower frequency (i.e., $50 \mathrm{~Hz}$ and $70 \mathrm{~Hz}$ ) bias tones $(p=0.0016)$. The median of the $150 \mathrm{~Hz}$ SFOAE bias data did not differ from the median of the lower frequency (i.e., $50 \mathrm{~Hz}$ and $70 \mathrm{~Hz}$ ) bias tone data.

The lower panel of Figure 8 shows the bias tone level that produced $50 \%$ CAP suppression in guinea pig as a function of tone-pip frequency. The CAP tone-pip frequencies were the same as the SFOAE probe frequencies. According to a linear regression analysis, the error measure did not vary significantly
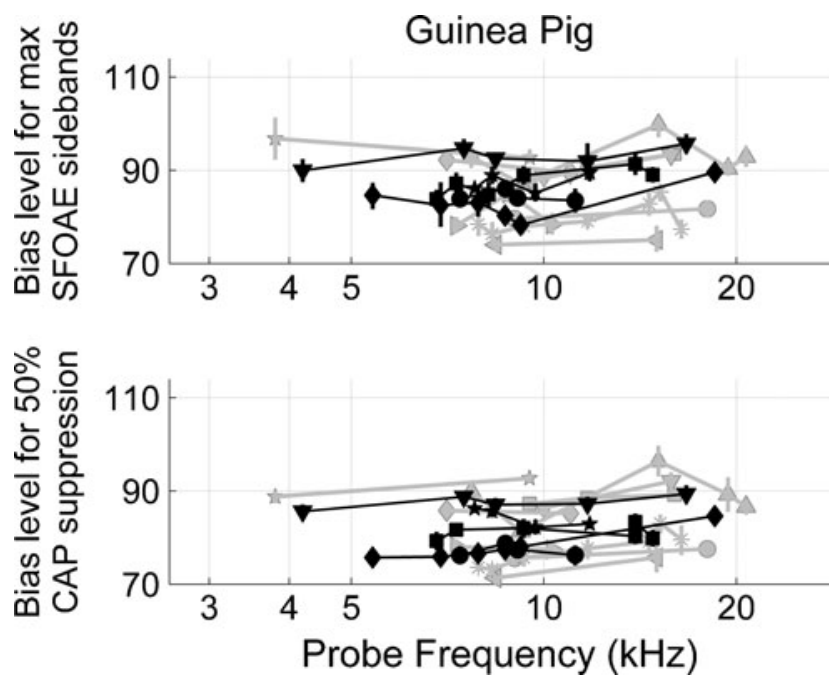

FIG. 8. The upper panel shows the bias level (dB SPL) that produced maximum SFOAE sidebands as a function of probe frequency. Vertical error bars are the width of the sideband versus bias level curves (e.g., Fig. 2) measured $0.5 \mathrm{~dB}$ down from the maximum. Data obtained with a $150 \mathrm{~Hz}$ bias tone are in gray (nine ears from eight guinea pigs) while data obtained with either a $50 \mathrm{~Hz}$ or $70 \mathrm{~Hz}$ bias tone are in black (five ears from five different guinea pigs). Different symbols represent different guinea pig ears. Lines connect data collected from a given ear. The lower panel shows the low-frequency bias tone level (dB SPL) needed to achieve 50\% CAP suppression as a function of frequency. Vertical error bars are estimates of $40 \%$ and $60 \%$ CAP suppression. Unlike the cat data, guinea pig data do not show a systematic trend with frequency.

with tone-pip frequency. Compared to the cat CAP data in Figure 7, guinea pig CAP data in Figure 8 required lower bias tone levels to yield maximum CAP suppression. As with guinea pig SFOAE data, the variance for $50 \mathrm{~Hz}$ and $70 \mathrm{~Hz}$ bias tone data was not statistically different $(p=0.0635)$. Data plotted in the same color did not yield significant results from the Levene's test of homoscedasticity. The variability among $150 \mathrm{~Hz}$ bias tone data differed from the variability among lower frequency (i.e., $50 \mathrm{~Hz}$ and $70 \mathrm{~Hz})$ bias tone data $(p=0.0036)$. The median of the $150 \mathrm{~Hz}$ CAP bias data was statistically indistinguishable from the median of the lower frequency bias data.

\section{Relating SFOAEs and CAPs}

For cat data, Figure 9 shows the relation between the bias level yielding maximum SFOAE sidebands and the bias level yielding 50\% CAP suppression. That is to say, Figure 9 shows the data in the upper panel of Figure 7 regressed on the data in the lower panel of Figure 7. The vertical SFOAE error bars in Figure 9 are the same as in the upper panel of Figure 7 and the horizontal CAP error bars are the same as the vertical error bars in the lower panel of Figure 7. The vertical error bars are barely noticeable on the scale of the lower panel of Figure 7 though are clearly seen in Figure 9. Linear regression analysis showed that the 


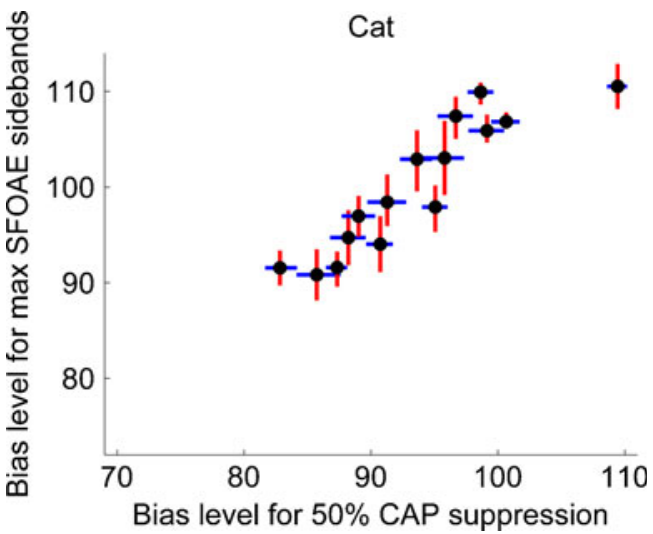

FIG. 9. The bias level (dB SPL) needed to achieve maximum SFOAE sidebands as a function of bias level needed to achieve $50 \%$ CAP suppression. The vertical error bars are the width of the SFOAE sideband versus bias level function (i.e., same as in the upper panel of Fig. 7). Likewise, the horizontal error bars are estimates of $40 \%$ and $60 \%$ CAP suppression (i.e., same as in the lower panel of Fig. 7). This is the relation we interpret to suggest that cat SFOAE and CAPs are both generated near the peak region of the travelling wave.

positive correlation had a slope of 0.92 , a $y$-intercept of 13.84, and a Pearson correlation coefficient of $r=$ 0.91 .

Figure 10 shows the relation between the bias level yielding maximum SFOAE modulation and the bias level yielding 50\% CAP suppression for guinea pig data; i.e., the data in the upper panel of Figure 8 regressed on the data in the lower panel of Figure 8. The vertical SFOAE error bars in Figure 10 are the same as in the upper panel of Figure 8 and the horizontal CAP error bars are the same as the vertical error bars in the lower panel of Figure 8. Linear

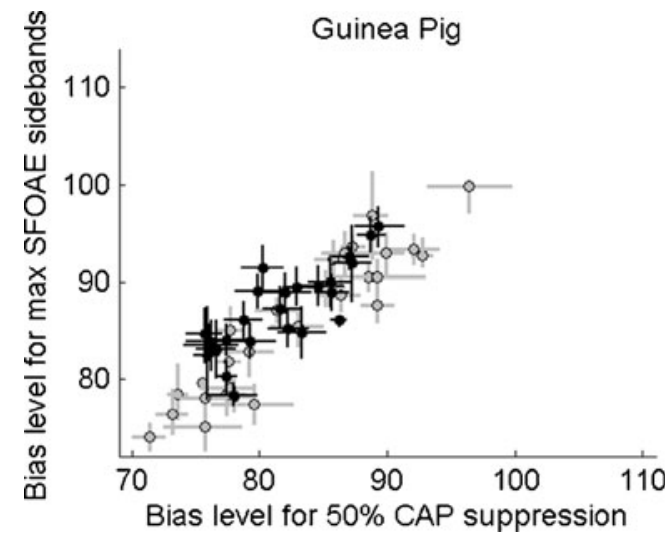

FIG. 10. The bias level (dB SPL) needed to achieve maximum SFOAE sidebands as a function of bias level needed to achieve $50 \%$ CAP suppression. The vertical error bars are the widths of the SFOAE sideband versus bias level function (i.e., same as in the upper panel of Fig. 8). The horizontal error bars are estimates of $40 \%$ and $60 \%$ CAP suppression (i.e., same as in the lower panel of Fig. 8). Gray data are from $150 \mathrm{~Hz}$ biasing while black data are from $50 \mathrm{~Hz}$ and $70 \mathrm{~Hz}$ biasing. This relation is what we believe illustrates that guinea pig SFOAE and CAPs are generated in the same cochlear place-the traveling wave peak region. regression analysis showed that the positive correlation for $150 \mathrm{~Hz}$ biasing (in gray) had a slope of 1.00, $y$ intercept of 2.69, and a Pearson correlation coefficient of $r=0.93$ while for lower frequency (i.e., $50 \mathrm{~Hz}$ and $70 \mathrm{~Hz}$ ) biasing (black) the slope was 0.89 , the $y$ intercept was 21.08, and the Pearson correlation coefficient $r=0.81$.

\section{Stability}

Figure 11 shows bias levels needed to produce maximum SFOAE sidebands for two cats (red) and two guinea pigs (black). Although these data show what was presented above-greater bias level was needed for maximum SFOAE sidebands in cats than in guinea pigs - the primary utility of these repeated measures was to use the variation through time to define what was "stable" and what was "unstable". The data in Figure 11 were recorded in the presence of several (e.g., two to six) probe tones and one bias tone that varied in level. Over the duration of about 6 to $8 \mathrm{~h}$, the variation in cat was minimal compared to the variation of bias level for maximum SFOAE sidebands as a function of probe frequency as seen in Figure 7. In contrast, the bias level needed for maximum SFOAE sidebands in guinea pigs varied by roughly 8 to $9 \mathrm{~dB}$ over the duration of time when these measures were made. From this, we conclude that cat cochleae were stable and guinea pig cochleae were unstable in the experimental conditions that were used.

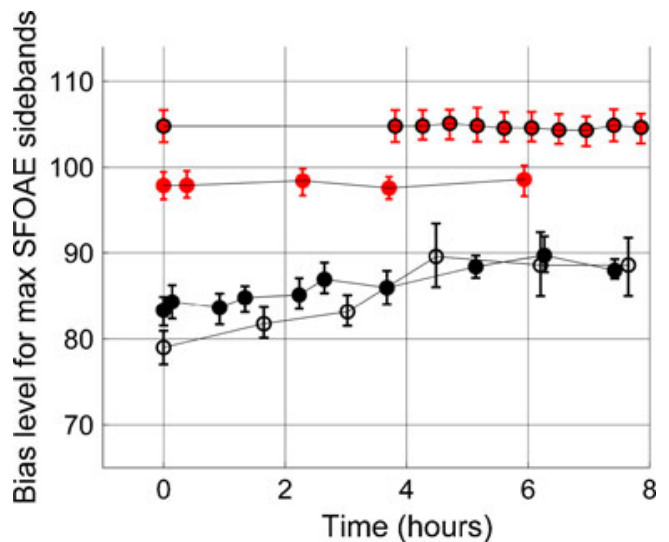

FIG. 11. Bias level (dB SPL) needed to produce maximum SFOAE sidebands as a function of time for two different cats (red) and two different guinea pigs (black). The probe tones were $9.091 \mathrm{kHz}$ (black edge) and $6.399 \mathrm{kHz}$ (no black edge) for the cats and $6.799 \mathrm{kHz}$ (filled symbol) and $7.898 \mathrm{kHz}$ (non-filled symbol) for the guinea pigs. The vertical error bars indicate the width of the sideband versus bias level function measured $0.5 \mathrm{~dB}$ down from the maximum. The cats show very little (i.e., less than $1 \mathrm{~dB}$ ) variation throughout the duration when these measures were made. In contrast, the guinea pig data varied by roughly 8 to $9 \mathrm{~dB}$. For reasons yet to be understood, cat cochleae were stable using our surgical preparation and our experimental protocol, though guinea pig cochleae were not stable. 


\section{DISCUSSION}

SFOAEs have been used to estimate the sharpness of cochlear tuning, to understand how emissions propagate out of the cochlea, and to test models of emission generation. Despite this progress, controversies remain. One controversy regards the latency of SFOAEs. Siegel et al. (2005) and Ruggero and Temchin (2007) suggested that SFOAE latencies are shorter than that predicted by Zweig and Shera's (1995) model of SFOAE generation. The most recent development of the latency controversy is that Shera et al. (2008) showed how discrepant latencies can be largely resolved if low-frequency SFOAEs are unmixed into their putative components that interfere with one another-short-latency emissions arising from placefixed generators and long-latency emissions arising from wave-fixed generators. A second controversy regards frequency tuning. Ruggero and Temchin (2005) argued that SFOAE-based tuning estimates do not accurately relate to cochlear tuning estimates as Shera et al. (2002) had suggested. The most recent development of the tuning controversy is that Shera et al. (2010) showed how SFOAE-based tuning estimates do not rely on direct basilar membrane measures (e.g., forward propagation delay), which was used as principal support for Ruggero and Temchin's (2005) argument. These controversies have roots in an unresolved question of where along the cochlea SFOAEs are generated, which itself is yet a third controversy. The work presented here addresses this third controversy. Interpretations of the results presented here do not rely on how SFOAEs propagate out of the cochlea or the accuracy of theories on how SFOAEs are generated.

Evidence for SFOAE generation near the peak region of the traveling wave

Low-frequency biasing levels needed to achieve criterion modulation of SFOAEs and CAPs were measured. A strong and positive trend between the bias level needed to modulate both SFOAEs and CAPs was found. Since it is well understood that CAPs are dominated by neural activity at the characteristic frequency place of the stimulus - the peak region of the traveling wave - we conclude that the majority of SFOAE generation is in a similar cochlear region. Particularly, results show that both SFOAEs and CAPs evoked from a $40 \mathrm{~dB}$ SPL probe exhibit strong modulation from similar low-frequency bias tone levels. If SFOAEs are a direct correlate of basilar membrane vibration, then the most credible interpretation of SFOAEs and CAPs showing similar sensitivity to low-frequency biasing is that both measures arise from excitation at similar cochlear places.
Our results support the hypothesis that SFOAEs are generated near the peak region. In contrast, our results do not offer support for Siegel et al. (2005) who compared forward and reverse propagation times and proposed that SFOAEs generated near the peak region are "deemphasized" compared to those generated in the tail region. The cause for the discrepancy between our results and those from Siegel et al. (2005) is not fully understood though could perhaps be, in part, because our SFOAE and neural measures were obtained from the same ears close in time while Siegel et al.'s (2005) were not. As reviewed in the "Introduction", both the SFOAE and neural measures used by Siegel et al. (2005) have high inter- and intraear variability.

How much of the total SFOAE generation occurs in the tail region of the traveling wave and how much occurs near the peak region? As part of their modeling effort, Choi et al. (2008) attempted to address this question. SFOAEs of non-peak origin were estimated by removing SFOAE generators spanning the width of their model's traveling wave peak region. Their calculation suggested that SFOAEs from the peak region were "comparable" [p. 2665 of Choi et al. (2008)] to those from the non-peak regions. Choi et al. (2008) made their calculation for only a $1 \mathrm{kHz}$ SFOAE - a frequency region where SFOAE generation is influenced by cochlear mechanics that are not yet fully understood (Shera et al. 2008, 2010). We cannot say if our empirical results do or do not offer support for Choi et al.'s (2008) modeling demonstration, as it is difficult to obtain reliable CAP measures below about $2 \mathrm{kHz}$ or so.

Here we consider three hypotheses to narrate our approach to the question of how much total SFOAE generation is near the peak and tail of the traveling wave. Each hypothesis interprets the slope of the linear function relating SFOAE and CAP biasing (i.e., Figs. 9 and 10).

1. If $100 \%$ of SFOAE generation was near the peak region, the slope relating SFOAE and CAP biasing would be $1 \mathrm{~dB} / \mathrm{dB}$. For every increase in bias level needed to achieve CAP suppression at different probe frequencies, an equivalent increase in bias level would be needed for SFOAE biasing at the same probe frequencies. Such a relation would indicate that SFOAE and CAP sites of generation are perfectly matched.

2. If $100 \%$ of an SFOAE was generated in the tail region, there would not be a slope to measure in Figures 9 and 10 because the expectation is that low-frequency biasing would have no effect on our otoacoustic emission measures. Since the action of two-tone suppression paradigms is in regions where there is saturation (Geisler et al. 1990), or non- 
linearity more specifically (Lukashkin and Russell 1998), the presently available interpretation of bias tone mechanisms (e.g. Cai and Geisler 1996) works only near the traveling wave peak regions where there is cochlear amplification-not in the tail region where there is no cochlear amplification. The hypothesis for net tail region generation to the total SFOAE is highly unlikely.

3. If SFOAE generation was partially near the peak region and partially in the tail region, and we continue to accept the sound interpretation that biasing acts only near the peak region of a probe tone's traveling wave described in hypothesis 2 , the slope relating SFOAE and CAP biasing would be less than $1 \mathrm{~dB} / \mathrm{dB}$. A slope of say $0.5 \mathrm{~dB} / \mathrm{dB}$, for example, would mean that for every 1-dB increase in bias level needed for CAP suppression, half of $1 \mathrm{~dB}$ bias level increase would be needed to bias the peak region's net contribution to the total SFOAE. The simple interpretation for the $0.5 \mathrm{~dB} /$ $\mathrm{dB}$ hypothetical example would be that $50 \%$ of the SFOAE was generated near the peak and the remaining $50 \%$ was from tail region generation that cannot be modulated by a bias tone.

The results from the present study yield slopes that are closest to the $1 \mathrm{~dB} / \mathrm{dB}$ hypothesis: $0.92 \mathrm{~dB} / \mathrm{dB}$ for cat and an average of $0.95 \mathrm{~dB} / \mathrm{dB}$ for the guinea pig bias groups. The simplest quantitative interpretation of these results is that cat and guinea pig SFOAEs evoked from commonly used $40 \mathrm{~dB}$ SPL probe levels are dominated by generation from the peak regions of the traveling wave. However, this study suffers from the same limitations as Brass and Kemp (1993), Siegel et al. (2005), and Keefe et al. (2008) in that we cannot determine precisely how much of the total SFOAE is generated near the peak and tail regions of the traveling wave.

\section{Species-dependent stability}

Criteria bias levels in guinea pigs did not show a rising trend with increasing probe frequency. Cats, in contrast, did show the rising trend. Results from repeated measures of SFOAE biasing (Fig. 11) suggest that one possible source of the inter-species difference was due to guinea pig cochleae being less stable than cat cochleae. We found this instability in the presence of tone-pip CAP threshold that stayed constant throughout the experiments. Guinea pig instability might only be true for the experimental preparation and protocol (i.e., anaesthesia, surgical approach, artificial ventilation, length of experiment, etc.) used for these experiments and may not be all that telling about the possibility of guinea pig cochlear instability in other studies. Then again, others have reported within- (Patuzzi and Moleirinho 1998; Zou et al. 2006) and between- (Sirjani et al. 2004; Brown et al. 2009) variability of guinea pig in vivo operating point estimates - a measure similar to the symmetry of our model transducer function.

To consider whether instability was the basis of the inability to detect a rising trend of our guinea pig data of Figure 8, an experiment was performed where multiple probe tones were simultaneously biased. Results in Figure 12 show that simultaneously biasing multiple probes reveals the trend that lower probe frequencies required less bias level. The graded effect was not measureable over the time course needed to obtain single-tone measures throughout the cochlea (i.e., Fig. 8) because instability caused the rising trend in Figure 12 to move up and down the $y$-axis. Nonetheless, the strong and positive relationships in Figure 10 were still present because both SFOAEs and CAPs were measured during the same moment of cochlear stability, thus allowing the guinea pig data to be regressed on one another to determine if the measures are generated in similar cochlear regions. The experimental paradigm used for Figure 12 was not used for SFOAE and CAP comparisons because we wanted the two measures to be recorded with similar paradigms. It is not possible to simultaneously bias CAPs from multiple tone-pip frequencies.

We presently do not have an explanation for the instability that is inherent to our guinea pig preparation and procedures and do not know its origin. Be that as it may, a question generated by the results of these experiments is if human cochleae are stable or instable in their experimental state, which is tremendously different than the highly invasive preparation used for cats and guinea pigs.

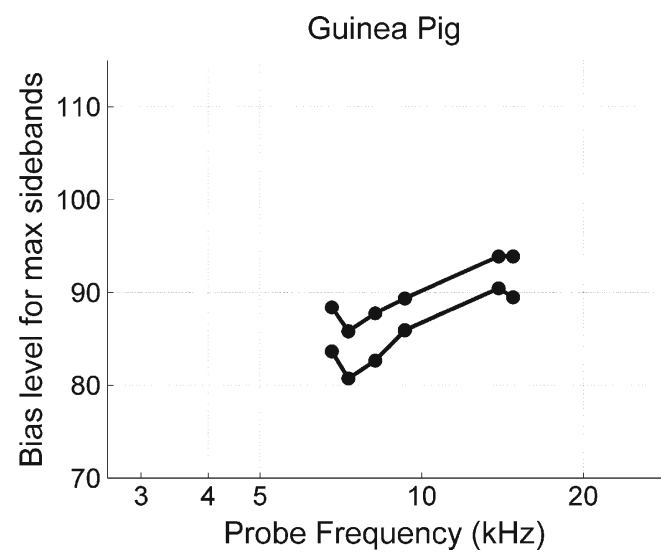

FIG. 12. Bias level (dB SPL) needed to produce maximum SFOAE sidebands as a function of probe tone frequency. On two different instances separated by approximately $4 \mathrm{~h}$ and $13 \mathrm{~min}$, we simultaneously presented six probe tones along with a bias tone that varied in level. The set of data with lower $y$-axis values was recorded first. This allowed us to see that guinea pig cochleae show the trend seen in the cat data-higher bias levels needed for higher probe frequencies-for each moment in time. 
Other reports of modulation effects on reflection-source emissions

SFOAE modulation in the present study was quantified by the level of the first-order sidebands at the probe frequency \pm the bias frequency. The amplitude of SFOAE sidebands varied with bias tone level (e.g., Fig. 5). These findings are consistent with Bian and Watts (2008) who described the influence of lowfrequency bias level on human SOAEs-anotherreflection source otoacoustic emission that is essentially an SFOAE that continuously self-evokes (Shera 2003). In their report, Bian and Watts (2008) showed how human SOAEs, as well as their sidebands, rise and then fall with increasing bias tone level. To the extent that SFOAEs and SOAEs are similarly generated, Bian and Watts' (2008) description of modulation of both SOAEs and their sidebands offers support for our interpretation that sidebands are indices of cochlear amplifier gain modulation.

Thus far, we have limited our consideration of suppression-based SFOAE experiments to those by Brass and Kemp (1993) and Keefe et al. (2008). Although interpretations remain unsettled, meeting abstracts by Siegel et al. (2003, 2004, 2005) and Shera et al. (2004) utilized suppression paradigms to determine where along the cochlea SFOAEs are generated. Siegel et al.'s (2003, 2004, 2005) empirical investigation lead them to conclude that doubt is cast on the hypothesis that generators near the peak region dominate SFOAEs. Shera et al. (2004) explored the suppression paradigm in a nonlinear active cochlear model and argued that the Siegel et al.'s $(2003,2004,2005)$ interpretation did not provide a valid determination of spatial locations of SFOAE generators along the cochlear partition. While previous suppression-based experiments aimed at determining place of SFOAE generation arrived at their conclusions though the use of suppressors primarily on the high-frequency side of the probe tone, experiments reported here used a much lower frequency bias tone. Low-frequency biasing paradigms probably do not produce additional SFOAE sources-mechanical perturbations - as do suppression paradigms.

\section{CONCLUSIONS}

Low-frequency bias tones were used to modulate SFOAEs and auditory nerve CAPs in both cats and guinea pigs. There was systematic variation of bias effects along the cochlea for cat ears though not guinea pig ears. Guinea pigs were found to be unstable over the many hours needed to collect data throughout their cochleae. Despite instability, guinea pig data could be used to determine where SFOAEs are generated because SFOAEs and CAPs from a given probe frequency were recorded closely in time. The strong and positive relationship for the bias level needed for maximal SFOAE modulation as a function of bias level needed for CAP suppression suggests that these two responses originate from similar cochlear regions - the peak of the traveling wave. These results do not offer support for the hypothesis that tail region generators are "emphasized" more than peak region generators in the total of an SFOAE.

\section{ACKNOWLEDGMENTS}

I thank John J. Guinan Jr. and Christopher A. Shera for their guidance through all stages of this project. For their valuable comments on the manuscript, I thank Maria A. Berezina, Nikolas A. Francis, Adam C. Furman, Ann E. Hickox, and three anonymous reviewers. This work was supported by grants F32 DC010112, RO1 DC00235, R01 DC03687, and P30 DC005209 from the NIDCD, National Institutes of Health.

\section{REFERENCES}

Bentsen T, Harte JM, DAU T (2011) Human cochlear tuning estimates from stimulus-frequency otoacoustic emissions. J Acoust Soc Am 129:3797-3807

Bian L, WATts KL (2008) Effects of low-frequency biasing on spontaneous otoacoustic emissions: amplitude modulation. J Acoust Soc Am 123:887-898

Brass D, KEMP DT (1993) Suppression of stimulus frequency otoacoustic emissions. J Acoust Soc Am 93:920-939

Brown DJ, Hartsock JJ, Gill RM, Fitzgerald HE, Salt AN (2009) Estimating the operating point of the cochlear transducer using low-frequency biased distortion products. J Acoust Soc Am 125:2129-2145

CAI Y, GEISLER CD (1996) Suppression in auditory-nerve fibers of cats using low-side suppressors. III. Model results. Hear Res 96:126-140

Cheatham MA, NaIk K, Dallos P (2010) Using the cochlear microphonic as a tool to evaluate cochlear function in mouse models of hearing. J Assoc Res Otolaryngol 12:113-125

Chertoff ME, Yi X, Lichtenhan JT (2003) Influence of hearing sensitivity on mechano-electric transduction. J Acoust Soc Am 114:3251-3263

Choi Y-S, Lee S-Y, Parham K, Neely ST, Kim DO (2008) Stimulusfrequency otoacoustic emission: measurements in humans and simulations with an active cochlear model. J Acoust Soc Am 123:2651-2669

Cody AR, Robertson D, Bredberg G, Johnston BM (1980) Electrophysiological and morphological changes in the guinea pig cochlea following mechanical trauma to the organ of Corti. Acta Otolaryngol 89:440-452

Dolan TG, Mills JH, Schmiedt RA (1985) A comparison of brainstem, whole-nerve AP and single-fiber tuning curves in gerbil: normative data. Hear Res 17:259-266

Geisler CD, Yates GK, Patuzzi RB, Johnstone BM (1990) Saturation of outer hair cell receptor currents causes two-tone suppression. Hear Res 44:241-56

Géléoc GS, Lennan GW, Richardson GP, Kros CJ (1997) "A quantitative comparison of mechanoelectrical trandsduction in 
vestibular and auditory hair cells of neonatal mice. Proc Biol Sci 264(1381):611-621

HudsPeth AJ, CoRey DP (1977) Sensitivity, polarity, and conductance changes in the response of vertebrate hair cells to controlled mechanical stimuli. Proc Natl Acad Sci USA 74:2407-2411

Keefe DH, Ellison JC, Fitzpatrick DF, Gorga MP (2008) Two-tone suppression of stimulus frequency otoacoustic emissions. J Acoust Soc Am 123:1479-1494

Kemp DT, Chum RA (1980) Observations on the generator mechanism of stimulus frequency acoustic emissions-two tone suppression. In: Brink GVD, Bilsen FA (eds) Psychophysical physiological and behavioral studies in hearing. Delft University Press, Delft, pp 34-42

KIANG NYS (1965) Discharge patterns of single-fibers in the cat's auditory nerve. In: M.I.T. research monograph no. 35. MIT Press, Cambridge

Klis JFL, Smoorenburg GF (1985) Modulation at the guinea pig round window of summating potentials and compound action potentials by low-frequency sound. Hear Res 20:15-23

KuIS JFL, Smoorenburg GF (1988) Cochlear potentials and their modulation by low-frequency sound in early endolymphatic hydrops. Hear Res 32:175-184

KonRad-Martin D, KefFe DH (2003) Time-frequency analyses of transient-evoked stimulus-frequency and distortion-product otoacoustic emissions: testing cochlear model predictions. J Acoust Soc Am 114:2021-2043

KonRad-Martin D, Keefe DH (2005) Transient-evoked stimulusfrequency and distortion-product otoacoustic emissions in normal and impaired ears. J Acoust Soc Am 117:3799-3815

Liberman MC (1984) Single-neuron labeling and chronic cochlear pathology. I. Threshold shift and characteristic frequency shift. Hear Res 16:33-41

Liberman MC (1990) Effects of chronic de-efferentation on auditorynerve response. Hear Res 49:209-224

LuKashKin AN, Russell IJ (1998) A descriptive model of the receptor potential nonlinearities generated by the hair cell mechanoelectrical transducer. J Acoust Soc Am 103:973-980

MeEnderink WF, van der Heijden M (2010) Reverse cochlear propagation in the intact cochlea of the gerbil: evidence for slow traveling waves. J Neurophysiol 103:1448-1455

Moleti A, Sisto R (2003) Objective estimates of cochlear tuning by otoacoustic emission analysis. J Acoust Soc Am 113:423-429

MoLETI A, Sisto R (2008) Comparison between otoacoustic and auditory brainstem response latency supports slow backward propagation of otoacoustic emissions. J Acoust Soc Am 123:1495-1503

Moleti A, Sisto R, Tognola G, Parazzini M, Ravazzani P, Grandori F (2005) Otoacoustic emission latency, cochlear tuning, and hearing functionality in neonates. J Acoust Soc Am 118:1576-1584

Patuzzi RB, Moleirinho A (1998) Automatic monitoring of mechanoelectrical transduction in the guinea pig cochlea. Hear Res 125:1-16

PatuzZi RB, Yates GK, Johnstone BM (1989) Outer hair cell receptor current and sensorineural hearing loss. Hear Res 42:47-72

Ruggero MA, Temchin AN (2005) Unexceptional sharpness of frequency tuning in the human cochlea. Proc Natl Acad Sci USA 102(51):18614-18619
Ruggero MA, Temchin AN (2007) Similarity of traveling-wave delays in the hearing organs of humans and other tetrapods. J Assoc Res Otolaryngol 8:153-166

Salt AN, Brown DJ, Hartsock JJ, PlontKe SK (2009) Displacement of the organ of Corti by gel injections into the cochlear apex. Hear Res 250:63-75

Schairer KS, Ellison JC, Fitzpatrick D, Keefe DH (2006) Use of stimulus-frequency otoacoustic emission latency and level to investigate cochlear mechanisms in human ears. J Acoust Soc Am 120:901-914

Sellick PM, Patuzzi R, Johnstone BM (1982) Modulation of responses of spiral ganglion cells in the guinea pig cochlea by low frequency sound. Hear Res 7:199-221

Shera CA (2003) Mammalian spontaneous otoacoustic emissions are amplitude-stabilized cochlear standing waves. J Acoust Soc Am 114:244-262

Shera CA, Guinan JJ (2003) Stimulus-frequency-emission group delay: a test of coherent reflection filtering and a window on cochlear tuning. J Acoust Soc Am 113:2762-2772

Shera CA, Guinan JJ, Oxenham AJ (2002) Revised estimates of human cochlear tuning from otoacoustic and behavioral measurements. Proc Natl Acad Sci USA 99:3318-3323

Shera CA, Tubis A, Talmadge CL, Guinan JJ (2004) The dual effect of 'suppressor' tones on stimulus-frequency otoacoustic emissions. Assoc Res Otolaryngol Abstr 27:538

Shera CA, Tubis A, Talmadge CL (2008) Testing coherent reflection in chinchilla: auditory-nerve responses predict stimulus-frequency emissions. J Acoust Soc Am 124:381-395

Shera CA, Guinan JJ, Oxenham AJ (2010) Otoacoustic estimation of cochlear tuning: validation in the chinchilla. J Assoc Res Otolaryngol 11:343-365

Siegel JH, Temchin AN, Ruggero MA (2003) Empirical estimates of the spatial origin of stimulus-frequency otoacoustic emissions. Assoc Res Otolaryngol Abstr 26:679

Siegel JH, Cerka AJ, Temchin AN, Ruggero MA (2004) Similar twotone suppression patterns in SFOAEs and the cochlear microphonics indicate comparable spatial summation of underlying generators. Assoc Res Otolaryngol Abstr 27:539

Siegel JH, Cerka AJ, Recio-Spinoso A, Temchin AN, van Dijk P, RugGero MA (2005) Delays of stimulus-frequency otoacoustic emissions and cochlear vibrations contradict the theory of coherent reflection filtering. J Acoust Soc Am 118:2434-2443

Sirjani DB, Salt AN, Gill RM, Hale SA (2004) The influence of transducer operating point on distortion generation in the cochlea. J Acoust Soc Am 115:1219-1229

Sisto R, Moleti A (2007) Transient evoked otoacoustic emission latency and cochlear tuning at different stimulus levels. J Acoust Soc Am 122:2183-2190

WeIss TF, LeONG R (1985) A model for signal transmission in an ear having hair cells with free-standing stereocilia. IV. Mechanoelectric transduction stage. Hear Res 20:175-195

Zou Y, Zheng J, Ren T, Nuttall A (2006) Cochlear transducer operating point adaption. J Acoust Soc Am 119:2232-2241

ZweIG, SHerA CA (1995) The origin of periodicity in the spectrum of evoked otoacoustic emissions. J Acoust Soc Am 98:2018-2047 